\title{
Factors Predictive of Remission of New-Onset Anterior Uveitis
}

\author{
Pichaporn Artornsombudh, MD ${ }^{1,2}$, Maxwell Pistilli, MS ${ }^{2}$, C. Stephen Foster, MD $^{1,3}$, \\ Siddharth S. Pujari, MBBS, MS ${ }^{1,4}$, Sapna S. Gangaputra, MD, MPH ${ }^{5,6}$, Douglas A. Jabs, MD, \\ MBA $^{7,8,9}$, Grace A. Levy-Clarke, MD ${ }^{10}$, Robert B. Nussenblatt, MD, MPH ${ }^{10}$, James T. \\ Rosenbaum, MD ${ }^{11,12}$, Eric B. Suhler, MD, MPH ${ }^{11,13}$, Jennifer E. Thorne, MD, PhD ${ }^{9,14}$, and \\ John H. Kempen, MD, PhD $2,15,16$
}

\begin{abstract}
${ }^{1}$ The Massachusetts Eye Research and Surgery Institution, Cambridge, Massachusetts ${ }^{2}$ Department of Ophthalmology, The Scheie Eye Institute, The Perelman School of Medicine, University of Pennsylvania, Philadelphia, Pennsylvania ${ }^{3}$ Department of Ophthalmology, Harvard Medical School, Boston, Massachusetts ${ }^{4}$ Om Eye Care Hospital, Belgaum, Karnataka, India ${ }^{5}$ Department of Surgery, Duke University School of Medicine, Durham, North Carolina ${ }^{6}$ Department of Ophthalmology and Visual Sciences, Fundus Photograph Reading Center, University of Wisconsin School of Medicine, Madison, Wisconsin ${ }^{7}$ Department of Ophthalmology, The Icahn School of Medicine at Mount Sinai, New York, New York ${ }^{8}$ Department of Medicine, The Icahn School of Medicine at Mount Sinai, New York, New York ${ }^{9}$ Department of Epidemiology, Bloomberg School of Public Health, The Johns Hopkins University, Baltimore, Maryland ${ }^{10}$ Laboratory of Immunology, National Eye Institute, National Institutes of Health, Bethesda, Maryland ${ }^{11}$ Department of Ophthalmology, Oregon Health and Science University, Portland, Oregon ${ }^{12}$ Department of Medicine, Oregon Health and Science University, Portland, Oregon ${ }^{13}$ Portland Veteran's Affairs Medical Center, Portland, Oregon ${ }^{14}$ Department of Ophthalmology, The Johns Hopkins University School of Medicine, Baltimore, Maryland ${ }^{15}$ Department of Ophthalmology, Center for Preventive Ophthalmology and Biostatistics, The Perelman School of Medicine, University of Pennsylvania, Philadelphia, Pennsylvania ${ }^{16}$ Department of Biostatistics and Epidemiology, Center for Clinical Epidemiology and Biostatistics, The Perelman School of Medicine, University of Pennsylvania, Philadelphia, Pennsylvania
\end{abstract}

\section{Abstract}

Purpose-To identify factors predictive of remission of inflammation in new-onset anterior uveitis cases treated at tertiary uveitis care facilities.

Design-Retrospective cohort study.

Participants-Patients seeking treatment at participating academic uveitis clinics within 90 days of initial diagnosis of anterior uveitis.

\footnotetext{
(C) 2013 by the American Academy of Ophthalmology

Correspondence: John H. Kempen, MD, PhD, Department of Ophthalmology, Center for Preventive Ophthalmology and Biostatistics, University of Pennsylvania, 3535 Market Street, Suite 700, Philadelphia, PA 19104. kempen@uphs.upenn.edu..

Presented at: 8th International Symposium on Uveitis, 2012, Sani, Greece. Financial Disclosure(s):

The author(s) have made the following disclosure(s):

C. Stephen Foster - Consultant - Eyegate, Allergan, Bausch \& Lomb, Sirion; Lecturer - Allergan, Bausch \& Lomb, Alcon, Inspire, Ista, Centocor

Douglas A. Jabs - Consultant - Roche, Genzyme Corporation, Allergan, Glaxo Smith Kline, The Emmes Corporation, The Johns Hopkins Dana Center for Preventive Ophthalmology; Data and Safety Monitoring Committee - Novartis, Applied Genetic Technologies Corporation

James T. Rosenbaum - Consultant - Abbott, ESBATech, Lux Biosciences, Centocor, Genentech; Equity owner - Amgen

John H. Kempen - Consultant - Lux Biosciences, Allergan, Alcon, Can-Fite, Clearside, Xoma.
} 
Methods-Retrospective cohort study based on standardized chart review.

Main Outcome Measures-Factors predictive of remission (no disease activity without corticosteroid or immunosuppressive treatments at all visits during a 90-day period).

Results-Nine hundred ninety eyes (687 patients) had a first-ever diagnosis of anterior uveitis within 90 days before initial presentation and had follow-up visits thereafter. The median followup time was 160 days. Systemic diagnoses with juvenile idiopathic arthritis (JIA; adjusted hazard ratio [aHR], 0.38; 95\% confidence interval [CI], 0.19-0.74) and Behçet's disease (aHR, 0.10; 95\% CI, 0.01-0.85) were associated with a lower incidence of uveitis remission. Cases of bilateral uveitis (aHR, $0.68 ; 95 \% \mathrm{CI}, 0.54-0.87$ ) and those with a history of cataract surgery before presentation (aHR, $0.51 ; 95 \%$ CI, 0.29-0.87) also had a lower incidence of remission. Regarding clinical findings at the initial visit, a high degree of vitreous cells at initial presentation was associated with a lower incidence of remission (for $1+$ or more vs. none: aHR, 0.72; 95\% CI, $0.55-0.95$ ). An initial visual acuity of 20/200 or worse, with respect to $20 / 40$ or better, also was predictive of a lower incidence of remission (aHR, 0.52; 95\% CI, 0.32-0.86).

Conclusions-Factors associated with a lower incidence of remission among new-onset anterior uveitis cases included diagnosis with JIA, Behçet's disease, bilateral uveitis, history of cataract surgery, findings of $1+$ or more vitreous cells at presentation, and an initial visual acuity of 20/200 or worse. Patients with these risk factors seem to be at higher risk of persistent inflammation; reciprocally, patients lacking these factors would be more likely to experience remission. Patients with risk factors for nonremission of uveitis should be managed taking into account the higher probability of a chronic inflammatory course.

Uveitis is a major cause of vision loss worldwide, reportedly accounting for 5\% to $20 \%$ of cases of legal blindness in the United States and Europe. ${ }^{1-4}$ The incidence of uveitis in the United States is estimated to be between 26 and 52 cases per 100000 persons per year. ${ }^{5,6}$ Anatomic classification of the site of uveitis activity divides uveitis into anterior uveitis, intermediate uveitis, posterior uveitis, and panuveitis. ${ }^{7}$ Anterior uveitis is the most common of these, ${ }^{8,9}$ diagnosed based on inflammation limited to the anterior portion of the eye (effecting the iris, ciliary body, or both). ${ }^{7,10}$ In cases of anterior uveitis, sometimes inflammation spills into the retrolental vitreous, in which case the term iridocyclitis may be used. $^{7}$

The clinical course of anterior uveitis can be characterized either by remission (sometimes followed by intermittent relapses) or by a chronic pattern, in which uveitis activity is persistent unless suppressed by anti-inflammatory therapy, recurring promptly when such therapy is discontinued. ${ }^{10}$ Sometimes the latter group eventually remits as well; clinical impression suggests that some patients may fit in one category at certain points and in the other category at other points in their clinical course. An expert consensus group suggested using 3 months of uveitis inactivity absent use of suppressive medication as indicative of a remission of uveitis. ${ }^{10}$

Anterior uveitis has a better visual prognosis than other types of uveitis but still can result in ocular complications such as cataract, glaucoma, band keratopathy, and posterior synechiae. ${ }^{3,11}$ Corticosteroid treatment often is effective in mitigating inflammation, but it also can lead to complications, particularly cataract and elevated intraocular pressure. The goal of treatment is to suppress inflammation and, if possible, to achieve remission of uveitis, thus avoiding the risk of further ocular injury as a result of uveitis or its treatment. ${ }^{12}$

Presently there is limited information available to predict whether a patient with new-onset anterior uveitis is likely to enjoy remission of the disease or to continue to experience ongoing ocular inflammation. Identification of factors predictive of remission would be useful to forecast the prognosis of the case and to guide the clinical management of such 
patients. Herein, we report the factors associated with medication-free remission of uveitis in a large cohort of eyes of patients who sought tertiary uveitis care within the first 90 days after an initial diagnosis of anterior uveitis.

\section{Methods}

The design of the Systemic Immunosuppressive Therapy for Eye Diseases Cohort Study has been described previously. ${ }^{13}$ The Systemic Immunosuppressive Therapy for Eye Diseases Cohort Study is a retrospective cohort study of patients with ocular inflammatory eye diseases seen at 5 tertiary referral centers in the United States. Only patients with noninfectious uveitis were included in this study, and patients with human immunodeficiency virus (HIV) or AIDS were excluded. The project was conducted in accordance with the principles of the Declaration of Helsinki, with the approval of the governing institutional review boards of each institution, each of which granted a waiver of consent that allows all living and deceased patients to be included.

All patients in the cohort (100\% data from all 5 centers) having disease activity, receiving anti-inflammatory treatments, or both at the time of presentation to one of the participating tertiary centers and diagnosed as having anterior uveitis within no more than 90 days before presentation were identified. Only data from these patients who had received a first ever diagnosis of anterior uveitis were used in this analysis. Follow-up data from the study database were used for retrospective follow-up for incidence of remission. Here, remission is used to indicate no evidence of active uveitis after discontinuation of all treatment (medication-free remission). For this analysis, cases of Fuchs' heterochromic cyclitis were excluded.

\section{Data Collection}

Information on all patients with inflammatory eye disease was obtained from medical records and was entered into computer-based standardized data entry forms by trained reviewers, as described previously. ${ }^{13,14}$ Data were collected with quality control checks requiring the reviewer to correct the errors in real time to optimize data within the constraints of a retrospective chart review study.

Presenting characteristics studied as potentially predictive of remission included demographic characteristics of the patients, bilateral versus unilateral uveitis, smoking status, the presence or absence of systemic diseases coexisting with ocular inflammation, and diagnostic and clinical features of anterior uveitis. Clinical findings at the initial visit analyzed as potential predictors included level of inflammatory activity and ocular findings including band keratopathy, posterior synechiae, keratic precipitates, intraocular pressure, and the presence of macular edema.

\section{Main Outcome Measures}

The primary objective was to evaluate predictive factors for remission in new-onset anterior uveitis cases in a tertiary setting. Remission previously was defined as no disease activity without corticosteroid or immunosuppressive treatments at all visits over at least a 90-day period. ${ }^{10}$ Given that the clinical practice at the participating centers did not call for patients to return more than 90 days after initial incidence of remission to verify sustained quiescence, it is likely that many patients did not return within 90 days of the onset of remission, but rather returned much later at the time of a relapse. Therefore, as a primary analysis, we counted eyes that did not return for follow-up after 90 days, had 1 or more follow-up visits before 90 days, and had remained inactive without receiving suppressive 
medications at all of those visits, as being in remission. Incidence of remission was assessed and calculated as cases at risk per eye-year of follow-up.

\section{Statistical Analysis}

The incidence of remission was evaluated on the basis of hazard ratios and adjusted hazard ratios (aHRs; with 95\% confidence intervals [CIs]), which were generated using crude and multivariate Cox proportional hazards models with a robust sandwich estimate to account for correlation between the eyes of individual patients. ${ }^{15}$ The proportion with remission at specific points were evaluated by calculating the cumulative incidence estimated from the crude Cox regression hazard function, which allowed nonlinear remission rates and confidence intervals consistent with the hazard ratios and accounting for correlation between eyes of the same patient. Plots were created using Kaplan-Meier analysis. All statistical analyses were performed with SAS software version 9.3 (SAS Inc., Cary, NC).

\section{Results}

Nine hundred ninety eyes of 687 patients were identified as having anterior uveitis diagnosed for the first time within 90 days of initial presentation to the clinics and had active inflammation, were taking suppressive therapy, or both at the time of presentation. These were followed up for remission, as defined above, over 1636 eye-years (1149 person-years). The median follow-up time was 0.44 eye-years (interquartile range, $0.21-1.41$ eye-years). The median age was 39 years with a 25 th to 75 th percentile range (interquartile range) of 27 to 52 years. The male-to-female ratio was 4:6. Seventy percent of the patients were white.

In this tertiary referral population at centers that typically manage severe cases, the KaplanMeier estimate of medication-free remission within 12 months after diagnosis of anterior uveitis was $47.2 \%$ (95\% CI, 42.6\%-51.5\%). In the more conservative sensitivity analysis in which cases were not considered in remission unless they had been observed at visits spanning at least 90 days as being inactive and not taking suppressive medications, the Kaplan-Meier estimate of remission within 12 months of diagnosis was $27.9 \%$ (95\% CI, $23.4 \%-32.1 \%$ ). (Often the next follow-up visit after 90 days was 1 year or more later, when the patient returned for an annual follow-up check; many cases were missing such a confirmatory visit and hence these patients were not counted as being in remission.)

Factors predictive of a lower incidence of medication-free remission included systemic diagnosis with either juvenile idiopathic arthritis (JIA; aHR, 0.38; 95\% CI, 0.19-0.74; Table 1; Fig 1) or Behçet's disease (aHR, 0.10; 95\% CI, 0.01-0.85; see Table 2, a long version of Table 1 that includes nonsignificant covariates omitted from Table 1, available online at http://aaojournal.org). Presentation with bilateral uveitis (aHR, 0.68; 95\% CI, 0.54-0.87) and a history of cataract surgery before presentation also were associated with a lower incidence of remission (aHR, 0.51; 95\% CI, 0.29-0.87). Age younger than 18 years was associated with a lower incidence of remission in the crude analysis, but the incidence of remission was similar to that of other age groups after adjusting for JIA status and other factors.

Regarding clinical findings at the initial visit, patients with active inflammation (aHR, 0.87; 95\% CI, 0.64-1.18) and slightly active inflammation (aHR, 1.05; 95\% CI, 0.67-1.63) had a similar incidence of medication-free remission as those with inactive uveitis who were taking suppressive therapy at the time of initial presentation. The presenting anterior chamber cell grade also was not predictive of remission. However, a high degree of vitreous cells at initial presentation was associated with a lower incidence of remission (for $1+$ or more vs. lower grades; aHR, 0.72; 95\% CI, 0.55-0.95; Fig 2). In addition, eyes with a visual acuity of 20/200 or worse had a lower incidence of remission than those with better visual 
acuity (with respect to 20/40 or better; aHR, 0.52; 95\% CI, 0.32-0.86; Fig 3). Other clinical findings at the time of initial presentation-including the presence of band keratopathy (aHR, 0.86; 95\% CI, 0.32-2.35), iris synechiae (aHR, 0.97; 95\% CI, 0.75-1.26), and hypopyon (aHR, 0.82; 95\% CI, 0.26-2.60) - were not found to be associated with an altered incidence of remission after adjusting for other factors. The presence of large keratic precipitates (aHR, 0.72; 95\% CI, 0.46-1.12) and macular edema (aHR, 0.70; 95\% CI, 0.461.06) at the time of presentation tended to be associated with a lower incidence of remission, but the associations were not statistically significant $(P=0.14$ and $P=0.10$, respectively).

As a sensitivity analysis, we evaluated remission more conservatively, requiring that eyes be observed to be completely inactive while not taking any medications at visits spanning 90 days or more. The point of this analysis was to see whether risk factor associations differed in an important way when a very conservative definition of remission was used; however, risk factor associations were similar in this sensitivity analysis, without important differences in the pattern of association of predictive factors. A difference worth noting in the sensitivity analysis was that current smoking tended to have a stronger negative association with remission (aHR of 0.53 vs. hazard ratio of 0.73 in the primary analysis), providing a suggestion of a negative effect of smoking similar to that previously reported by another of our analyses of a broader ocular inflammatory diseases population. ${ }^{16}$

\section{Discussion}

The primary interest our results provide is with regard to factors predictive of medicationfree remission of primary anterior uveitis, which may provide a basis for counseling patients regarding the likely clinical course of their disease, as well as providing guidance for clinical management.

Juvenile idiopathic arthritis-associated uveitis has been reported to be the most common identifiable cause of uveitis in childhood. ${ }^{17}$ Among children with uveitis, a chronic clinical course was noted more often in JIA and predicted association with JIA. ${ }^{18}$ It has been stated that there are 2 major clinical presentations of uveitis in JIA, characterized by chronic anterior uveitis and acute anterior uveitis with recurrent attacks. ${ }^{19}$ Juvenile idiopathic arthritis-associated uveitis also reportedly can manifest as a biphasic course, showing a high initial disease activity, followed by a quiescence and then a recurrence of inflammation during puberty. ${ }^{20}$ However, there are reports demonstrating that $60 \%$ to $80 \%$ of the patients had an indolent chronic course lasting more than 3 months, ${ }^{18,21}$ in addition to several other reports finding that JIA-associated uveitis cases most often have persistent disease. ${ }^{22-24}$ Our findings were consistent with these latter observations, indicating that JIA-associated anterior uveitis has a $62 \%$ lower incidence of remission than other cases of anterior uveitis reaching tertiary uveitis care. Given the relatively low overall incidence of remission among anterior uveitis cases receiving tertiary uveitis care, this observation suggests that an initial plan to embark on chronic suppressive therapy may be justified in most settings for this condition.

Behçet's disease-associated anterior uveitis classically presents with a relapsing iridocyclitis persisting for up to 3 months ${ }^{25,26}$ and may run a chronic relapsing course, not always resolving completely between attacks. ${ }^{26}$ Although Behçet's disease-associated anterior uveitis was infrequent in our study, the incidence of remission was exceptionally low. It is possible that the known severity of this condition may have made the clinicians more likely to treat the condition with suppressive therapy, perhaps leading to an underestimate of the incidence of remission. However, it is unlikely that this led to a 10 -fold difference in the risk, suggesting that the probability of remission lasting 3 months or more may be low, at least in the first few years, with Behçet's disease-associated anterior uveitis. These 
observations, along with the severity of the condition, suggest that initially prescribing suppressive therapy also may be justified in most settings for Behçet's disease-associated anterior uveitis.

Regarding other systemic disease associations, we did not find significant associations with the incidence of remission. Many of these were infrequent in the analysis population, making it difficult to rule out an association with the available evidence. It is also possible, in a retrospective ophthalmic chart review study such as this one, that certain nonocular attributes or diagnoses may have been undercaptured, leading to nondifferential misclassification that could blunt associations with incident remission. In particular, HLAB27 status-which was known to be positive in only $17 \%$ of the anterior uveitis caseslikely was underascertained, because it may not have been tested for routinely in the earlier years of the cohort. We chose to omit this covariate from our report because its lack of association with incidence in remission in this analysis (data not shown) likely was on the basis of underascertainment. With nondifferential misclassification of this nature, positive associations observed should be reliable, but negative associations may reflect missed associations resulting from misclassification. Therefore, our design is less robust to rule out associations than to rule them in. Fortunately, given that both JIA-associated uveitis and particularly Behçet's disease have a central ocular component, it is unlikely that the magnitude of these associations was severely underestimated.

Regarding presenting characteristics of cases of primary anterior uveitis, cases with bilateral disease had a significantly lower risk of remission than unilateral cases. Bilateral disease may reflect a greater degree of immune dysregulation, a greater degree of disease severity, or both, which may be associated with a greater likelihood of persistence of ocular inflammation.

There are several potential explanations for our observation that a history of cataract surgery was associated with a lower incidence of remission in anterior uveitis. Several reports describe acute anterior uveitis after cataract surgery sometimes resulting in persistent inflammation. ${ }^{27-29}$ Possible explanations of this phenomenon include inflammation related to retained lens material, uveitis related to surgical trauma, and intraocular lens-associated uveitis (iris chafing that can cause mechanical irritation and chronic inflammation). We were unable to investigate further the potential contributions of these possible mechanisms in our database because of its retrospective nature. However, it seems plausible that uveitis arising from some of these mechanisms would be prone to chronicity.

Anterior uveitis, although characterized by inflammation primarily in anterior segment of the eye, can be associated with vitreous inflammatory cells detectable on clinical examination. The concept of anterior and intermediate uveitis, ${ }^{10}$ a classification that had not been proposed at the time most patients in this study were treated, describes patients who have substantial inflammation both in the vitreous and anterior chamber. It is unclear whether there is a complete dichotomy between these 2 categories, or if there is some degree of continuum in the distribution of the site of inflammation between anterior and intermediate uveitis archetypes. Our results demonstrating a higher degree of vitreous cells (1+ or more) at presentation as predictive of a substantially lower incidence of remission seems consistent with the concept that cases with more attributes of intermediate uveitis are more likely to have persistent disease, even as intermediate uveitis in general has a lower incidence of remission than anterior uveitis. ${ }^{30,31}$ Another possible explanation of the observed pattern is that cases with more vitreous spillover are more severe and therefore are more likely to have persistent ocular inflammation. The increased severity explanation for increased risk of persistent uveitis is consonant with our observation that presenting visual 
acuity of 20/200 or worse was associated with the lower incidence of remission, which may be explained on the same basis.

The strengths of this study included a favorable sample size, increasing statistical precision, and the use of quality control methods to optimize the accuracy of retrospective data collection. The main limitation of our study is that it was a retrospective study conducted at tertiary uveitis centers. Incomplete follow-up and missing data, as well as the study of a tertiary population, likely led to an underestimation of the remission rate, such that the overall estimate of remission incidence is of limited generalizability, especially outside the tertiary setting. On average, more severe cases would be referred for tertiary care and tertiary care sometimes may taper treatment more slowly. Each of these factors would lead to a longer estimated time to remission. However, regarding the identification of factors predictive of (non)remission of primary anterior uveitis - the primary goal of the study-the probability of accurately identifying disease remission would have to have a strong tertiary center-factor interaction (large differences between tertiary and nontertiary cases in the effects of the factors under study) for the observed associations to be seen on the basis of bias. Such extreme interaction seems unlikely, particularly given that the associations observed are supported by pathogenetic theories and in some cases by prior observations. Furthermore, should such interactions exist, our results still should be generalizable to cases managed at tertiary centers. In addition, the number of events observed with some potential predictors of interest were relatively small despite the multicenter approach, leading to imprecise estimates of the incidence of remission with respect to these factors and leaving open the possibility that unusual conditions may be predictive of an altered incidence of remission that were not identified in this study because of type II error.

In summary, our study of cases of new-onset anterior uveitis treated at tertiary uveitis centers within 90 days of initial diagnosis identified several presenting factors associated with a lower incidence of medication-free remission, including systemic diagnosis with JIA or Behçet's disease, bilaterality of uveitis, a history of cataract surgery, a high degree of vitreous cells at initial presentation (1+ or more), and a presenting visual acuity of 20/200 or worse. Patients with these risk factors are probably at higher risk of persistent inflammation, particularly those with more than one of these factors. Reciprocally, patients lacking these factors would be more likely to experience remission. Patients with these potential risk factors for nonremission of uveitis should be educated and managed taking into account the higher probability of a chronic inflammatory course, which in turn may indicate closer monitoring or longer-term treatment of these cases, or both.

\section{Supplementary Material}

Refer to Web version on PubMed Central for supplementary material.

\section{Acknowledgments}

Supported by the National Eye Institute, National Institutes of Health, Bethesda, Maryland (grant no.: EY014943 [J.H.K.]); Research to Prevent Blindness, Inc., New York, New York; the Paul and Evanina Mackall Foundation, New York, New York; and the Ocular Immunology and Uveitis Foundation, Cambridge, Massachusetts. During the time of data collection, J.H.K. was an Research to Prevent Blindness James S. Adams Special Scholar Award recipient, J.E.T. was a Research to Prevent Blindness Harrington Special Scholar Award recipient, and D.A.J. and J.T.R. were Research to Prevent Blindness Senior Scientific Investigator Award recipients. G.A.L-C. is an employee of Johnson \& Johnson and was previously supported by the National Eye Institute, and R.B.N. continues to be supported by intramural funds of the National Eye Institute. E.B.S. receives support from the United States Department of Veterans' Affairs. None of the sponsors had any role in the design and conduct of the report; collection, management, analysis, and interpretation of the data; or in the preparation, review, and approval of this manuscript. 


\section{References}

1. Darrell RW, Wagener HP, Kurland LT. Epidemiology of uveitis. Incidence and prevalence in a small urban community. Arch Ophthalmol. 1962; 68:502-14. [PubMed: 13883604]

2. Nussenblatt RB. The natural history of uveitis. Int Ophthalmol. 1990; 14:303-8. [PubMed: 2249907]

3. Rothova A, Suttorp-van Schulten MS, Frits Treffers W, Kijlstra A. Causes and frequency of blindness in patients with intraocular inflammatory disease. Br J Ophthalmol. 1996; 80:332-6. [PubMed: 8703885]

4. Suttorp-Schulten MS, Rothova A. The possible impact of uveitis in blindness: a literature survey. $\mathrm{Br}$ J Ophthalmol. 1996; 80:844-8. [PubMed: 8962842]

5. Gritz DC, Wong IG. The incidence and prevalence of uveitis in northern California: the Northern California Epidemiology of Uveitis Study. Ophthalmology. 2004; 111:491-500. [PubMed: 15019324]

6. Suhler EB, Lloyd MJ, Choi D, et al. Incidence and prevalence of uveitis in Veterans Affairs Medical Centers of the Pacific Northwest. Am J Ophthalmol. 2008; 146:890-6. [PubMed: 19027424]

7. Bloch-Michel E, Nussenblatt RB. International Uveitis Study Group recommendations for the evaluation of intraocular inflammatory disease. Am J Ophthalmol. 1987; 103:234-5. [PubMed: 3812627]

8. Chang JH, Wakefield D. Uveitis: a global perspective. Ocul Immunol Inflamm. 2002; 10:263-79. [PubMed: 12854035]

9. London NJ, Rathinam SR, Cunningham ET Jr. The epidemiology of uveitis in developing countries. Int Ophthalmol Clin. 2010; 50:1-17. [PubMed: 20375859]

10. Standardization of Uveitis Nomenclature (SUN) Working Group. Standardization of uveitis nomenclature for reporting clinical data. Results of the First International Workshop. Am J Ophthalmol. 2005; 140:509-16. [PubMed: 16196117]

11. Durrani OM, Meads CA, Murray PI. Uveitis: a potentially blinding disease. Ophthalmologica. 2004; 218:223-36. [PubMed: 15258410]

12. Kruh J, Foster CS. The philosophy of treatment of uveitis: past, present and future. Dev Ophthalmol. 2012; 51:1-6. [PubMed: 22517200]

13. Kempen JH, Daniel E, Gangaputra S, et al. Methods for identifying long term adverse effects of treatment in patients with eye diseases: the Systemic Immunosuppressive Therapy for Eye Diseases (SITE) Cohort Study. Ophthalmic Epidemiol. 2008; 15:47-55. [PubMed: 18300089]

14. Kempen JH, Daniel E, Dunn JP, et al. Overall and cancer related mortality among patients with ocular inflammation treated with immunosuppressive drugs: retrospective cohort study. BMJ. 2009; 339:b2480. [PubMed: 19578087]

15. Liang KY, Zeger SL. Regression analysis for correlated data. Annu Rev Public Health. 1993; 14:43-68. [PubMed: 8323597]

16. Galor A, Feuer W, Kempen JH, et al. Adverse effects of smoking on patients with ocular inflammation. Br J Ophthalmol. 2010; 94:848-53. [PubMed: 20606023]

17. Smith JA, Mackensen F, Sen HN, et al. Epidemiology and course of disease in childhood uveitis. Ophthalmology. 2009; 116:1544-51. [PubMed: 19651312]

18. Heinz C, Mingels A, Goebel C, et al. Chronic uveitis in children with and without juvenile idiopathic arthritis: differences in patient characteristics and clinical course. J Rheumatol. 2008; 35:1403-7. [PubMed: 18484686]

19. Kanski JJ. Anterior uveitis in juvenile rheumatoid arthritis. Arch Ophthalmol. 1977; 95:1794-7. [PubMed: 303091]

20. Hoeve M, Kalinina Ayuso V, Schalij-Delfos NE, et al. The clinical course of juvenile idiopathic arthritis-associated uveitis in childhood and puberty. Br J Ophthalmol. 2012; 96:852-6. [PubMed: 22399692]

21. Marvillet I, Terrada C, Quartier P, et al. Ocular threat in juvenile idiopathic arthritis. Joint Bone Spine. 2009; 76:383-8. [PubMed: 19524473] 
22. Kotaniemi K, Arkela-Kautiainen M, Haapasaari J, Leirisalo-Repo M. Uveitis in young adults with juvenile idiopathic arthritis: a clinical evaluation of 123 patients. Ann Rheum Dis. 2005; 64:871-4. [PubMed: 15897307]

23. Grassi A, Corona F, Casellato A, et al. Prevalence and outcome of juvenile idiopathic arthritisassociated uveitis and relation to articular disease. J Rheumatol. 2007; 34:1139-45. [PubMed: 17343317]

24. Kalinina Ayuso V, van de Winkel EL, Rothova A, de Boer JH. Relapse rate of uveitis postmethotrexate treatment in juvenile idiopathic arthritis. Am J Ophthalmol. 2011; 151:217-22. [PubMed: 21145533]

25. Kitaichi N, Miyazaki A, Iwata D, et al. Ocular features of Behcet's disease: an international collaborative study. Br J Ophthalmol. 2007; 91:1579-82. [PubMed: 18024808]

26. Evereklioglu C. Current concepts in the etiology and treatment of Behçet disease. Surv Ophthalmol. 2005; 50:297-350. [PubMed: 15967189]

27. Kang HM, Park JW, Chung EJ. A retained lens fragment induced anterior uveitis and corneal edema 15 years after cataract surgery. Korean J Ophthalmol. 2011; 25:60-2. [PubMed: 21350698]

28. Pandit RT, Coburn AG. Sudden corneal edema due to retained lens nuclear fragment presenting 8.5 years after cataract surgery. J Cataract Refract Surg. 2011; 37:1165-7. [PubMed: 21596261]

29. Teo L, Chee SP. Retained lens fragment in the anterior segment as a cause of recurrent anterior uveitis. Int Oph-thalmol. 2010; 30:89-91.

30. Vidovic-Valentincic N, Kraut A, Hawlina M, et al. Intermediate uveitis: long-term course and visual outcome. Br J Ophthalmol. 2009; 93:477-80. [PubMed: 19066184]

31. Chan SM, Gan KD, Weis E. Characteristics and predictors of recurrence of anterior and intermediate uveitis in a Canadian referral centre. Can J Ophthalmol. 2010; 45:144-8. [PubMed: 20379299] 


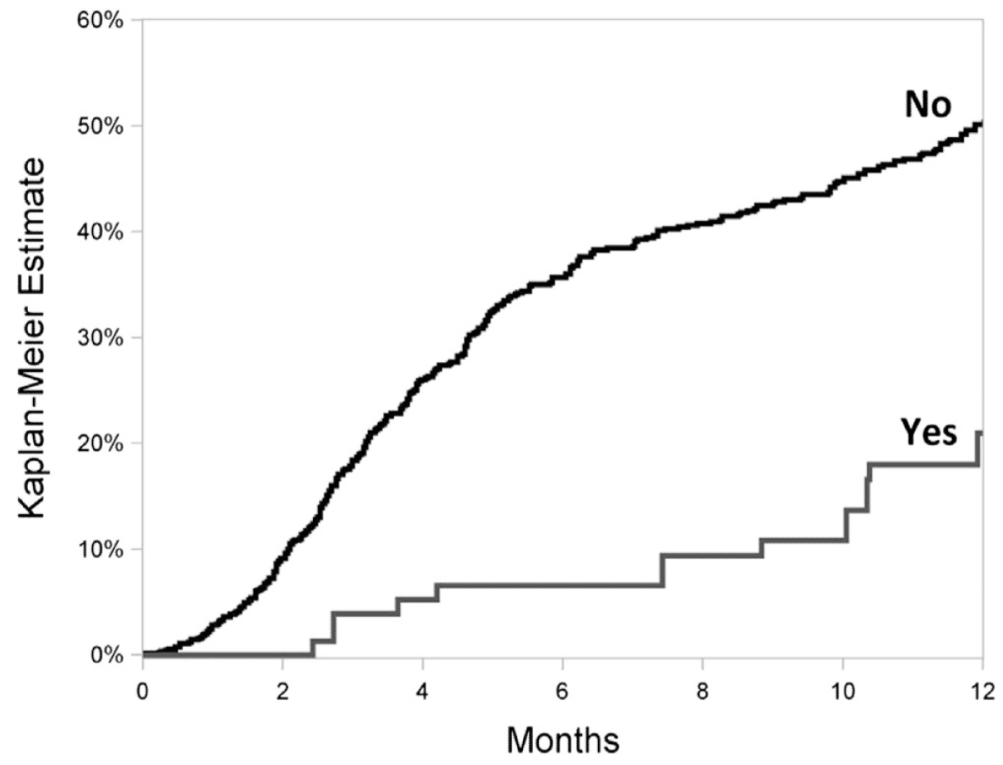

Figure 1.

Kaplan-Meier estimation of the incidence of remission in cases with and without a systemic diagnosis of juvenile idiopathic arthritis. 


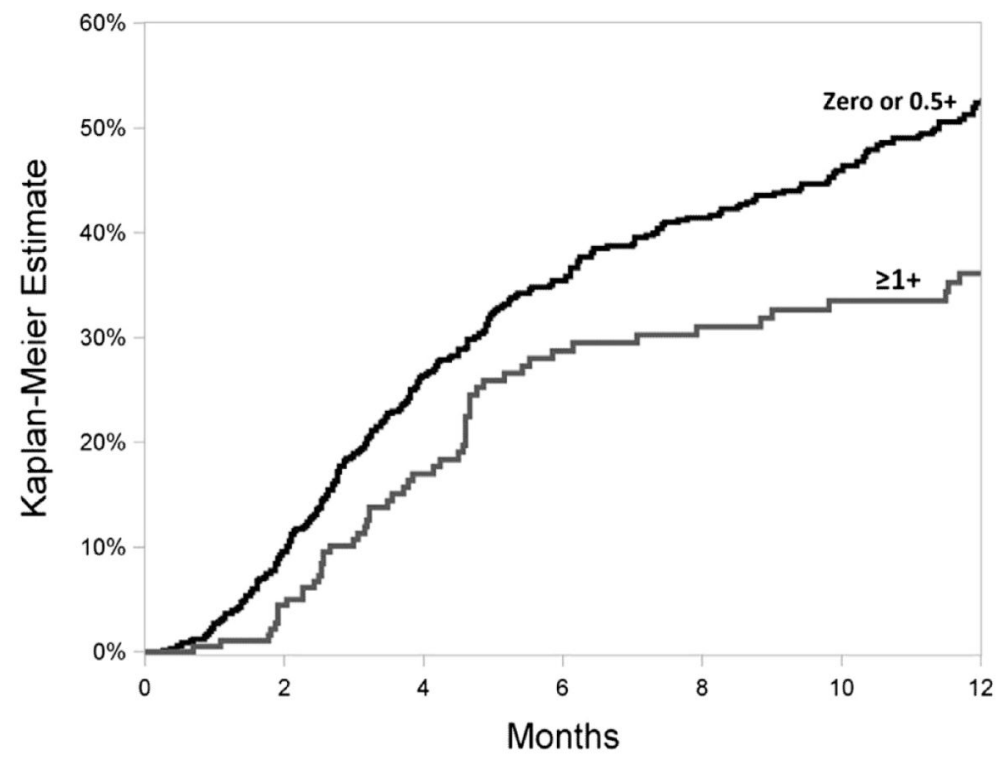

Figure 2.

Kaplan-Meier estimation of the incidence of remission of anterior uveitis by vitreous cell grade (none or $0.5+$ vs. $1+$ or worse) at the time of presentation. 


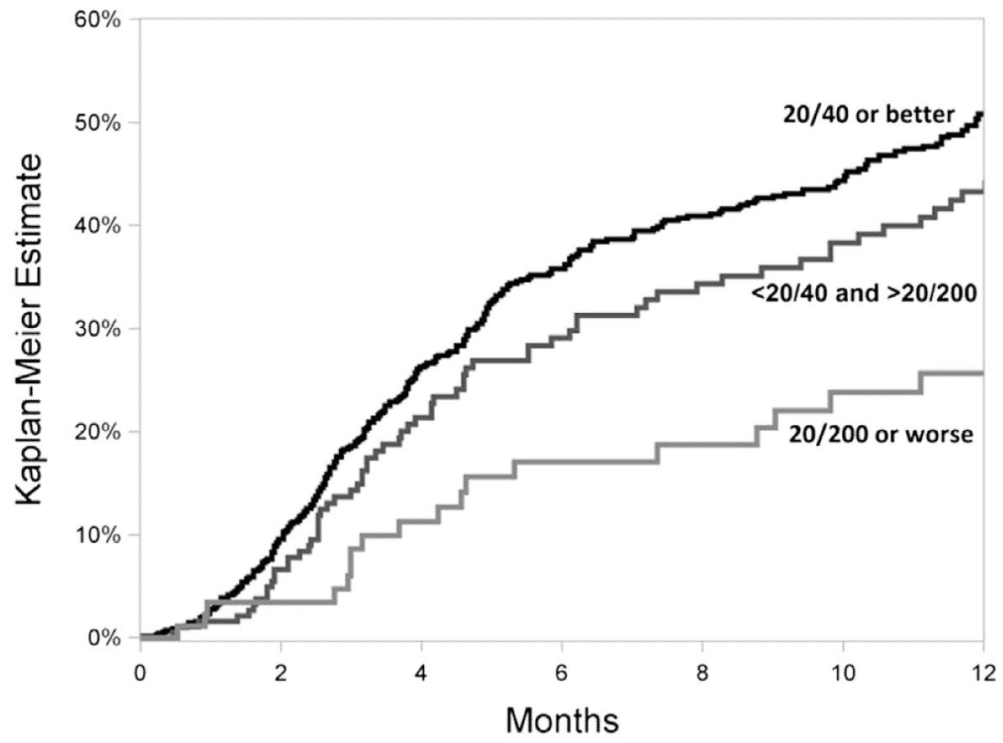

Figure 3.

Kaplan-Meier estimation of the incidence of remission of anterior uveitis by the level of presenting visual acuity. 
Table 1

Presenting Factors Potentially Predictive of Remission of Anterior Uveitis (Hazard Ratios Less Than 1 Are Unfavorable, Indicating a Lower Incidence of Remission)

\begin{tabular}{|c|c|c|c|c|}
\hline Characteristics at Presentation & $\begin{array}{c}\text { Crude Hazard Ratio } \\
\text { (95\% Confidence Interval) }\end{array}$ & $P$ Value & $\begin{array}{l}\text { Adjusted* Hazard Ratio } \\
\text { (95\% Confidence Interval) }\end{array}$ & $P$ Value \\
\hline \multicolumn{5}{|l|}{ Age (yrs) } \\
\hline$<18$ & 1.00 (reference group) & $<0.01$ & 1.00 & 0.35 \\
\hline $18-35$ & $1.61(1.13-2.29)$ & & $1.37(0.89-2.09)$ & \\
\hline $36-55$ & $1.81(1.30-2.52)$ & & $1.23(0.82-1.86)$ & \\
\hline$>55$ & $1.74(1.21-2.49)$ & & $1.48(0.93-2.35)$ & \\
\hline Bilateral uveitis & $0.72(0.59-0.88)$ & $<0.01$ & $0.68(0.54-0.87)$ & $<0.01$ \\
\hline \multicolumn{5}{|l|}{ Systemic disease/laboratory characteristics } \\
\hline Juvenile idiopathic arthritis & $0.39(0.26-0.60)$ & $<0.001$ & $0.38(0.19-0.74)$ & $<0.01$ \\
\hline Behçet's disease & $0.11(0.01-0.95)$ & 0.04 & $0.10(0.01-0.85)$ & 0.04 \\
\hline Prior cataract surgery & $0.49(0.31-0.78)$ & $<0.01$ & $0.51(0.29-0.87)$ & 0.01 \\
\hline \multicolumn{5}{|l|}{ Ocular findings } \\
\hline \multicolumn{5}{|l|}{ Visual acuity } \\
\hline $20 / 40$ or better & 1.00 (reference group) & $<0.001$ & 1.00 & 0.04 \\
\hline$<20 / 40$ and $>20 / 200$ & $0.85(0.67-1.08)$ & & $0.93(0.71-1.23)$ & \\
\hline $20 / 200$ or worse & $0.47(0.31-0.70)$ & & $0.52(0.32-0.86)$ & \\
\hline \multicolumn{5}{|l|}{ Overall inflammatory activity } \\
\hline Inactive & 1.00 (reference group) & 0.59 & 1.00 & 0.54 \\
\hline Slightly active & $1.12(0.74-1.68)$ & & $1.05(0.67-1.63)$ & \\
\hline Active & $0.94(0.71-1.23)$ & & $0.87(0.64-1.18)$ & \\
\hline \multicolumn{5}{|l|}{ Anterior chamber cell grade } \\
\hline Quiet & 1.00 (reference group) & 0.85 & 1.00 & 0.89 \\
\hline $0.5+$ & $1.18(0.85-1.65)$ & & $1.12(0.79-1.57)$ & \\
\hline $1+$ & $1.02(0.74-1.40)$ & & $0.95(0.67-1.35)$ & \\
\hline $2+$ & $1.09(0.80-1.49)$ & & $0.97(0.68-1.37)$ & \\
\hline \multicolumn{5}{|l|}{ Vitreous cell grade } \\
\hline Quiet/0.5+ & 1.00 (reference group) & 0.01 & 1.00 & 0.02 \\
\hline$\geq 1+$ & $0.72(0.55-0.93)$ & & $0.72(0.55-0.95)$ & \\
\hline Band keratopathy & $0.24(0.11-0.50)$ & $<0.001$ & $0.86(0.32-2.35)$ & 0.77 \\
\hline Iris synechiae & $0.68(0.54-0.86)$ & $<0.01$ & $0.97(0.75-1.26)$ & 0.81 \\
\hline
\end{tabular}

Ophthalmology. Author manuscript; available in PMC 2015 March 01. 